ARTICLE

\title{
Defect-driven antiferromagnetic domain walls in CuMnAs films
}

Sonka Reimers ${ }^{1,2 凶}$, Dominik Kriegner (1) ${ }^{3,4}$, Olena Gomonay (D) ${ }^{5}$, Dina Carbone (i) ${ }^{6}$, Filip Krizek ${ }^{4}$, Vit Novák (i) ${ }^{4}$, Richard P. Campion', Francesco Maccherozzi ${ }^{2}$, Alexander Björling6 ${ }^{6}$, Oliver J. Amin ${ }^{1}$, Luke X. Barton', Stuart F. Poole ${ }^{1}$, Khalid A. Omari ${ }^{1}$, Jan Michalička7, Ondřej Man (10 7, Jairo Sinova ${ }^{5}$, Tomáš Jungwirth (1) 1,4, Peter Wadley (iD ${ }^{1}$, Sarnjeet S. Dhesi (i) ${ }^{2 凶}$ \& Kevin W. Edmonds (iD) ${ }^{1 凶}$

Efficient manipulation of antiferromagnetic (AF) domains and domain walls has opened up new avenues of research towards ultrafast, high-density spintronic devices. AF domain structures are known to be sensitive to magnetoelastic effects, but the microscopic interplay of crystalline defects, strain and magnetic ordering remains largely unknown. Here, we reveal, using photoemission electron microscopy combined with scanning $X$-ray diffraction imaging and micromagnetic simulations, that the AF domain structure in CuMnAs thin films is dominated by nanoscale structural twin defects. We demonstrate that microtwin defects, which develop across the entire thickness of the film and terminate on the surface as characteristic lines, determine the location and orientation of $180^{\circ}$ and $90^{\circ}$ domain walls. The results emphasize the crucial role of nanoscale crystalline defects in determining the AF domains and domain walls, and provide a route to optimizing device performance.

\footnotetext{
${ }^{1}$ School of Physics and Astronomy, University of Nottingham, Nottingham NG7 2RD, UK. ${ }^{2}$ Diamond Light Source, Chilton OX11 ODE, UK. ${ }^{3}$ Institut für Festkörper- und Materialphysik and Würzburg-Dresden Cluster of Excellence ct.qmat, Technische Universität Dresden, 01062 Dresden, Germany. ${ }^{4}$ Institute of Physics, Czech Academy of Sciences, 16200 Praha 6, Prague, Czech Republic. ${ }^{5}$ Institut für Physik, Johannes Gutenberg Universität Mainz, 55099 Mainz, Germany. ${ }^{6}$ MAX IV Laboratory, Lund University, 22100 Lund, Sweden. ${ }^{7}$ Central European Institute of Technology, Brno University of Technology, 612

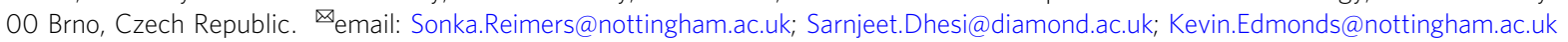


key goal of spintronics is the development of high-speed, high-density data storage devices that are robust against magnetic fields. Antiferromagnetic (AF) materials offer a route to realising these goals since they exhibit intrinsic dynamics in the THz-regime, lack magnetic stray fields, and can be electrically switched $^{1-4}$. Moreover, AF order is exhibited in a wide range of materials compatible with the properties of insulators, semiconductors, and metals. Electrical switching has been achieved in several AF systems, with the resulting current-induced domain modifications attributed to spin-orbit torques or thermomagnetoelastic effects ${ }^{4-7}$. Spin-orbit torque manipulation of $\mathrm{AF}$ domains was first achieved using orthogonal current pulses to induce $90^{\circ}$ rotations of the $\mathrm{AF}$ order parameter, but more recently current-polarity dependent switching of AF order has been achieved and ascribed to domain wall motion ${ }^{8}$. AF domains and domain walls are therefore the building blocks of AF spintronics, but pinning can limit device performance whilst creep affects long-term memory stability. In ferro- and ferrimagnets, magnetic domain formation has been extensively studied for decades and is well-known to be largely governed by the minimization of the demagnetizing field energy 9,10 . On the other hand, domain formation in fully compensated antiferromagnets remains largely unexplored.
Domain morphologies in AF thin films vary considerably with thickness and nanostructure shape which has been ascribed to strain effects, although evidence for a direct relationship is lacking ${ }^{11-15}$. To date, device concepts have considered an ideal AF spin lattice ${ }^{3,16,17}$, but high-resolution AF domain imaging has revealed pronounced non-uniformities and pinning effects during domain switching $5,6,8,18-20$.

Here, we show the relationship between magnetic domain structures and structural defects in the metallic antiferromagnet CuMnAs, which is a focus of AF spintronics research due to its favorable crystal symmetry for spin-orbit torque switching. In CuMnAs thin films, elongated microtwins and atomically sharp anti-phase boundaries ${ }^{21}$ have recently been identified as the most prominent defects. The anti-phase boundaries have been associated with atomically sharp $180^{\circ} \mathrm{AF}$ domain walls ${ }^{22}$. The microtwin defects, which are the focus of this work, are shown to have a dramatic influence on the AF domain configuration in CuMnAs thin films. We show that microtwin defects largely control the domain structure by generating pinned $90^{\circ}$ domain walls and confining $180^{\circ}$ domain walls.

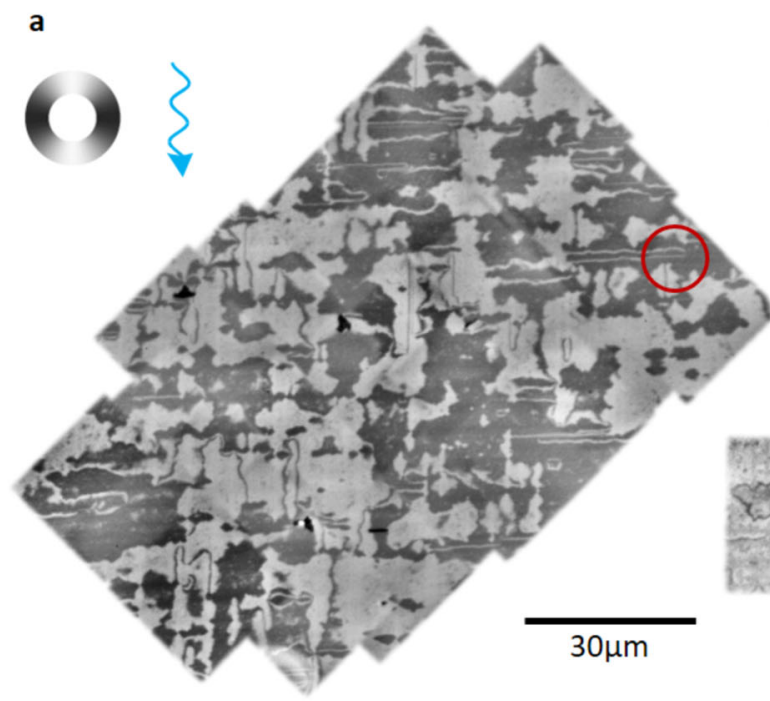

b
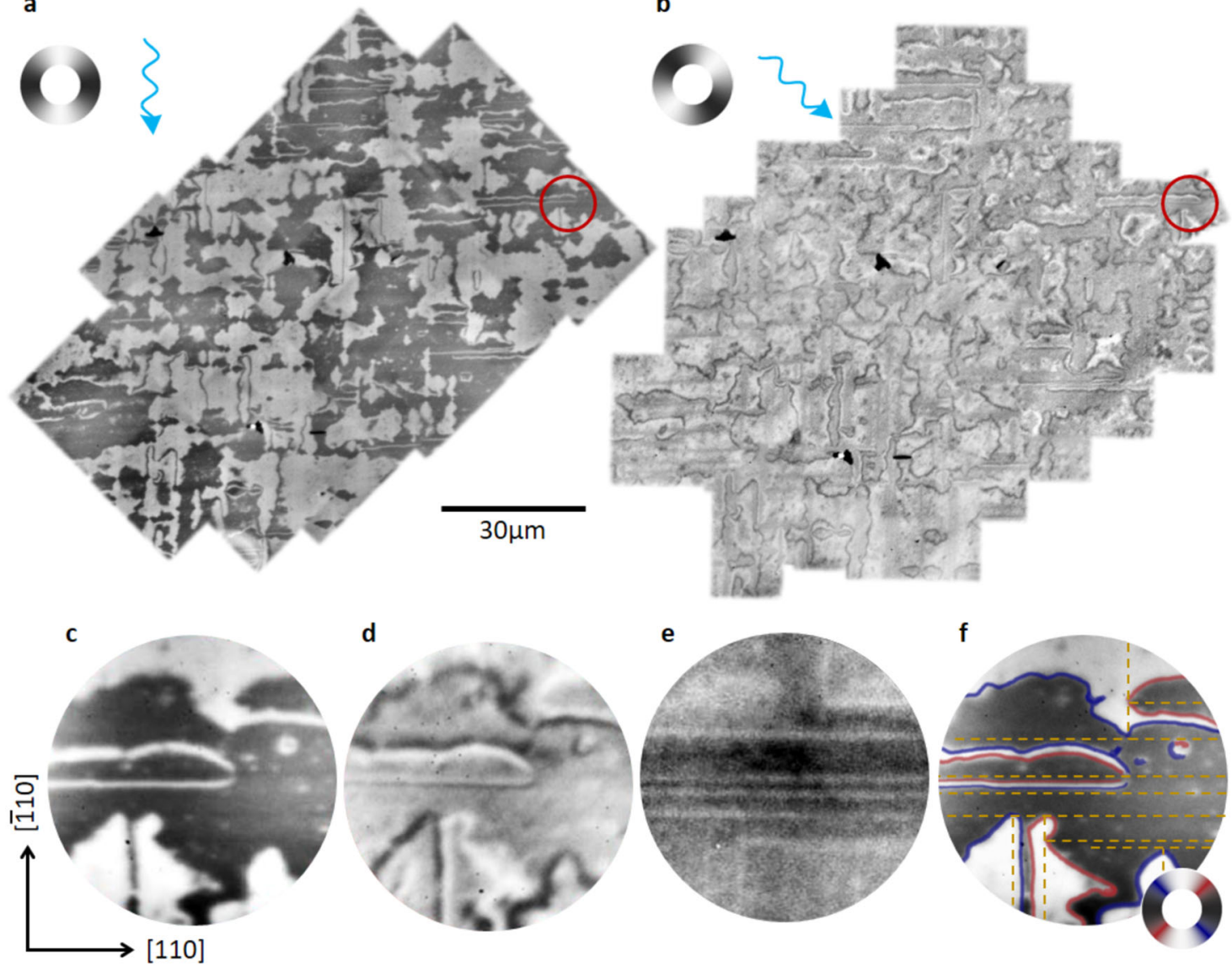

Fig. 1 AF domains and domain walls. a XMLD-PEEM image showing AF domains with spin axes parallel to [110] (dark areas) or [110] (light areas). b XMLD-PEEM image for the same area as (a) showing AF domain walls with spin axes parallel to [100] (black lines) or [010] (white lines); the contrast in the domains appears gray. The greyscale wheels indicate the local spin axis in each image and the blue arrows represent the incident $X$-ray direction. The diameter of the red circle is $12.5 \mu \mathrm{m}$. c High-resolution XMLD-PEEM image of the domains from the circled area in (a). d High-resolution XMLD-PEEM image of the domain walls from the circled area in (b). e High-resolution XLD-PEEM image of the same area as in (c) and (d) showing lines arising from defects. $\mathbf{f}$ Composite image showing the relationship between the XMLD-PEEM and XLD-PEEM images. The black and white areas show the magnetic domains, solid red and blue lines show the domain walls with the orientation indicated by the color wheel and the broken yellow lines represent the structural defects revealed by XLD-PEEM. 


\section{Results}

The 50-nm-thick CuMnAs(001) films were grown epitaxially on $\mathrm{GaP}(001)^{21}$. The CuMnAs layer is a collinear antiferromagnet with a Néel temperature of $485 \mathrm{~K}^{23}$ and a tetragonal crystal structure $(a=b=3.853 \AA, c=6.278 \AA)$. Close lattice-matching along the half-diagonal of the cubic GaP substrate unit cell ensures fully strained epitaxial growth with low mosaicity ${ }^{24}$. The spin axes align in the (001)-plane, i.e., in the plane of the film ${ }^{25}$ due to a strong magnetocrystalline anisotropy. In the following, the crystallographic axes refer to the orientation of the CuMnAs crystal.

The AF domain structure was imaged using high-resolution photoemission electron microscopy (PEEM) combined with $\mathrm{X}$-ray magnetic linear dichroism (XMLD) ${ }^{26}$. Figure $1 \mathrm{a}, \mathrm{b}$ shows large area maps imaged with the X-ray polarization vector (E) aligned to highlight the AF domain structure and domain boundaries, respectively. Maximum XMLD contrast is observed between regions with the local spin axis aligned perpendicular and parallel to $\mathbf{E}$. We observe approximately equal populations of light and dark areas in Fig. 1a, corresponding to domains with the local spin axis parallel to [110] or [110] (see Supplementary Note 1). Figure 1c, $d$ shows high-resolution XMLD-PEEM images of the red circled area in Fig. 1a and b. The AF domains typically exceed several $\mu \mathrm{m}$ in lateral size and generally have serrated edges.

The boundaries between the domains are visible in Fig. 1b, d, when $\mathbf{E}$ is at $45^{\circ}$ to the local spin axis in both AF domains. In this imaging configuration, the domains have the same contrast and the domain boundary contrast dominates. $90^{\circ}$ domain walls appear as well separated black or white lines, depending on the average direction of the spin axis across the domain wall, with typical width $(400 \pm 50) \mathrm{nm}$. Adjacent black and white lines in Fig. $1 \mathrm{~b}, \mathrm{~d}$ correspond to $180^{\circ}$ domain walls (see Supplementary Note 2).

X-ray Linear Dichroism (XLD) combined with PEEM is sensitive to local changes in the charge anisotropy and can therefore act as an indicator of local crystallographic variations, i.e., structural defects. Figure 1e shows an XLD-PEEM image of the

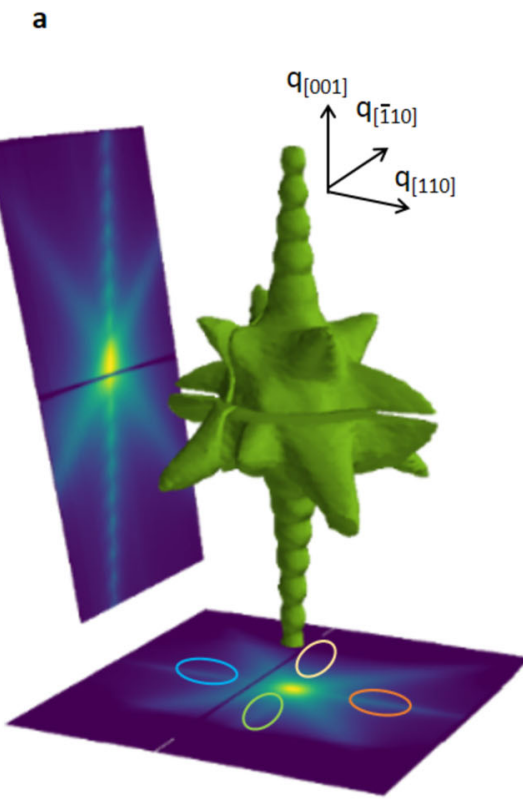

red circled area in Fig. 1a and b which reveals a pattern of thin lines running parallel to the [110] and [110] crystallographic directions. Figure if shows the AF domain structure superimposed with the domain wall contrast (blue and red lines) along with the structural defect pattern (broken yellow lines). Direct comparison of the XMLD-PEEM and XLD-PEEM images over the same area shows that the local AF spin axis is always oriented collinear with the defect lines.

Long straight $180^{\circ}$ domain walls are found to be confined between two parallel defects. These domain walls extend over several microns and can be seen as the long, thin light and dark lines in Fig. 1a. In some cases these domain walls become highly constricted between two neighboring defects as seen in the middle of Fig. 1f. The $90^{\circ}$ domain walls form corners in areas where two defects are orthogonal, as for example in the bottom half of Fig. 1f, which form the serrated edges seen in Fig. 1a.

Bulk-sensitive crystallographic information on the nature of defects in thin films, with nanoscale spatial resolution, can be achieved using scanning X-ray diffraction microscopy (SXDM ${ }^{27}$. Crystallographic defects lead to specific contributions to a reciprocal space map (RSM). A three-dimensional RSM of the CuMnAs (003) Bragg peak, generated from two-dimensional diffraction images for several sample tilts (see "Methods", Supplementary Note 3 and Supplementary Movie) is shown in Fig. 2a. The RSM has a modulated intensity along $q_{[001]}$ arising from the finite film thickness ${ }^{28}$ as well as strong diffuse scattering along the $q_{\langle 101\rangle}$-type directions, which has been attributed to antiphase boundaries along the $\{011\}$ planes $^{21}$. Sharper intensity streaks, hereafter referred to as wings, along the $q_{\langle 110\rangle}$-type directions indicate the presence of another type of defect. These wings are only visible for specific areas of the sample and are marked by the colored ovals in the lower panel of Fig. 2a.

Mapping the intensity of these wings generates real-space images of the defects. Maps produced using the intensity of one of the wings yield bright defect lines, on a homogeneous background, which run perpendicular to the direction of the selected wing, see Fig. 2b. The lines obtained from separate wings are complementary to each other, i.e., each wing produces a separate
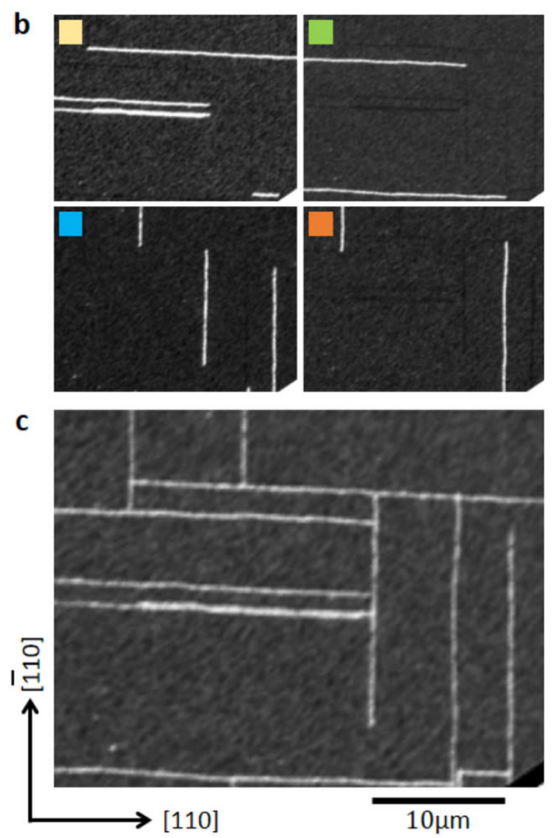

Fig. 2 Bulk microtwinning projected onto the CuMnAs film surface. a (003) RSM isosurface (green solid) and projections along the $q_{(110)}$ (left panel) and $q_{(001)}$ (bottom panel) planes. b Real-space SXDM maps extracted from the wings. The color code indicates the areas of reciprocal space used which are given by the corresponding colored ovals in (a). c Sum of the four images shown in (b). 
set of lines. The collated lines arising from all wings are given in Fig. 2c which reveals a rectangular pattern of defect lines running along the [110] and [110] directions, reminiscent of the pattern revealed by XLD-PEEM in Fig. 1e. The four complementary sets of defect lines with specific $q$-dependence of the scattering revealed by SXDM indicate defect orientations along four different crystallographic directions in the bulk, while the XLDPEEM images only show their surface termination.

High-angle annular dark field-scanning transmission electron microscopy (HAADF-STEM) reveals that the defects are slabs of a microtwinned phase, in which the lattice is rotated so that the $c$-axis is tilted away from the film normal by $\sim 82^{\circ}$, as shown in Fig. $3 \mathrm{a}, \mathrm{b}$. The slabs extend over most of the film thickness and grow wider towards the sample surface where they produce the characteristic rectangular pattern with lines running parallel to the [110] and [110] directions, Fig. 3c. Figure 3b shows a highresolution image of a microtwin defect where the atomic ordering is indicated. The microtwin and surrounding bulk film form a coherent boundary, with the microtwin slab extending along one of the $\{111\}$ planes. In particular, for each defect line on the surface there are two possible bulk defect slab orientations with opposite tilts $^{21}$ which can be distinguished in SXDM, but not in XLD-PEEM.

As the magnetic easy-plane in tetragonal CuMnAs is perpendicular to the $c$-axis ${ }^{25}$, the $\sim 82^{\circ}$ rotation of the $c$-axis in the microtwin region will have a profound effect on the local spin orientation. The microtwin region and surrounding bulk film share only one magnetic easy axis, determined by the intersection of the easy planes (purple sheets in Fig. 3c). This easy axis is represented by the orange line in Fig. 3c. For any microtwin defect line on the surface, there are two possible propagation directions into the bulk, but for either case the easy axis remains parallel to the defect line on the surface. The local Néel vectors (i.e., the difference in the sublattice magnetic moment directions) then aligns parallel to the microtwin surface termination. For adjacent microtwin defects, the local Néel vector can align either parallel or antiparallel. Antiparallel alignment results in the $180^{\circ}$ domain walls seen in the XMLD-PEEM images in Fig. 1. For parallel alignment of the Néel vector, the area between the microtwin defects is magnetically homogeneous and can extend over several microns. Perpendicular alignment of two defects gives rise to $90^{\circ}$ domain walls.

In the final part, we show that including the effects of microtwin defects in micromagnetic simulations is sufficient to fully explain the experimentally observed AF domain structures shown in Fig. 1. The simulations consider an AF layer with two (equivalent) orthogonal in-plane easy axes, i.e., without considering an out-of-plane variation of the Néel vector. The effects of microtwins are included as a rotation of the magnetocrystalline

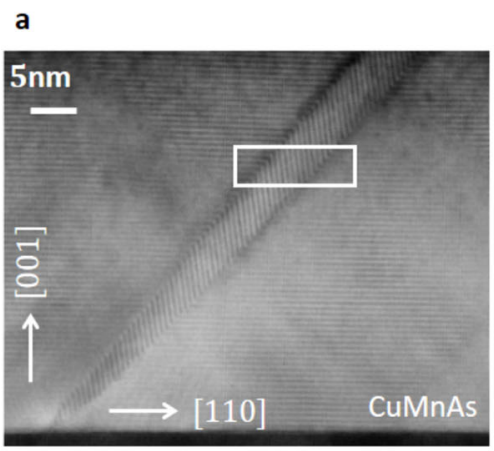

b

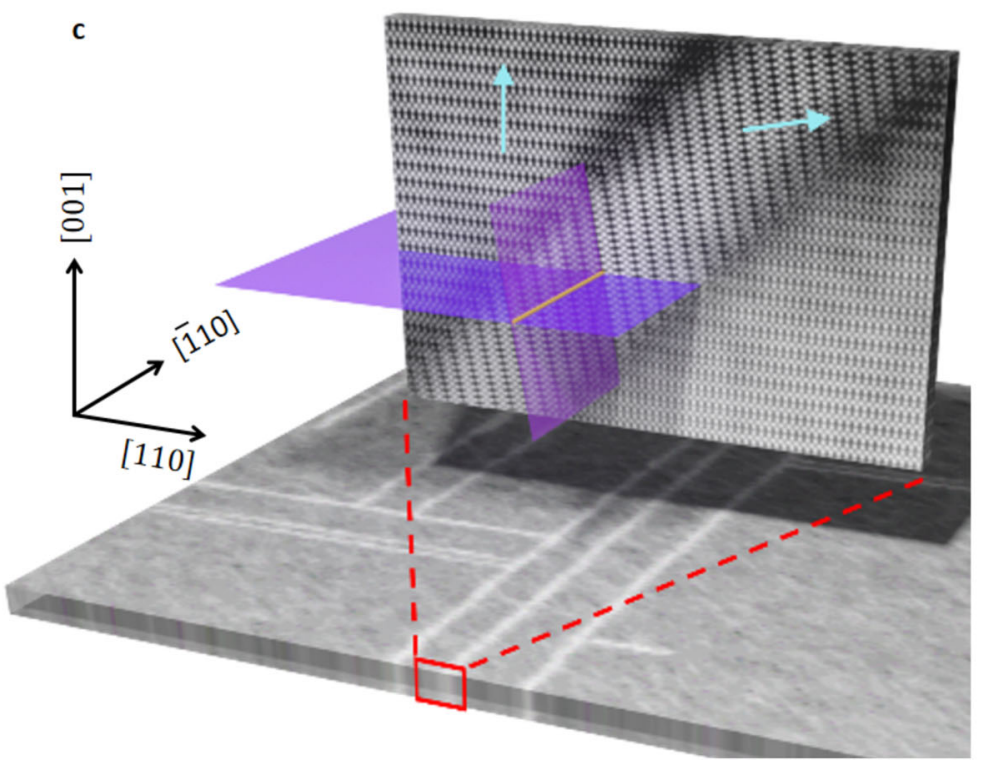

Fig. 3 Atomic structure of the microtwin defects. a HAADF-STEM image of a microtwin defect in a CuMnAs thin film. b HAADF-STEM image from the area shown by the white rectangle in (a), with an atomic model overlay. The microtwin and surrounding bulk film form a coherent boundary indicated by the gray line. c SXDM map of defect lines on the CuMnAs(001) surface (horizontal panel) and HAADF-STEM image (vertical plane) of a microtwin defect. Teal arrows give the local c-axis orientation. Purple sheets indicate the magnetic easy planes in the microtwin and the surrounding film. The orange line shows the intersection of the magnetic easy planes which determines the local spin axis for the microtwin and surrounding area. 
anisotropy localized at the microtwins along with a homogeneous strain field, perpendicular to the microtwins (see Supplementary Note 4). We consider that the extrinsic strain due to the microtwin is much larger than the spontaneous strains due to the magnetic texture, such that the inverse effect of the magnetic texture on the strain distribution ${ }^{29}$ can be neglected. The simulations show that the spin axis in the vicinity of a microtwin defect line is always aligned parallel to the defect line.

Figure $4 \mathrm{a}, \mathrm{b}$ shows the simulated $\mathrm{AF}$ domain structures for two parallel microtwin defect lines with a different spacing between the lines. For this configuration, antiparallel alignment of the Néel vectors on either side of the microtwin defects results in a $180^{\circ}$ domain wall. For large separations of the microtwins, the domain wall width is determined by the strain-induced magnetic anisotropy. For small separations, the rotated magnetocrystalline anisotropy of the microtwin defects leads to a narrower, highly confined domain wall. The simulations show a close resemblance to the XMLD-PEEM image of the $180^{\circ} \mathrm{AF}$ domain wall shown in the middle of Fig. 4e. The domain wall is narrow and straight in the confined area on the left of Fig. 4e whereas it becomes wider and meanders on the right of the image where the microtwin defects are much further apart.

Figure $4 \mathrm{c}, \mathrm{d}$ shows simulated AF domain structures for two parallel microtwin defect lines terminating on a perpendicular microtwin defect line for two different initial boundary configurations. For a parallel boundary configuration of the Néel vectors across the two parallel defect lines (Fig. 4c), the simulations converge to a homogeneous AF domain configuration across the defect lines (see lower half of Fig. 4c). On the other hand, a $90^{\circ}$ domain wall forms close to the perpendicular defect line with characteristic pinch points close to the $\mathrm{T}$-junctions reminiscent of the serrated edges seen on the AF domains in the experiment (Fig. 4f). A similar result is found for an antiparallel initial boundary configuration, but with an additional $180^{\circ}$ domain wall between the parallel defect lines. This $180^{\circ}$ domain wall unzips into two $90^{\circ}$ domain walls with opposite senses of rotation along with pinch points at the T-junctions. The simulation closely reproduces the AF configuration imaged using XMLD-PEEM (Fig. 4g).

\section{Discussion}

The microscopic AF domain structure in CuMnAs thin films has been shown to be dominated by microtwin defects that appear as lines along specific crystallographic directions on the film surface. The local spin axis is aligned parallel to the defect lines. This then leads to either large $\mathrm{AF}$ domains with serrated edges or $180^{\circ}$ domain walls running parallel to the [110] or [110] crystallographic directions. A perpendicular orientation of two microtwin defect lines leads to $90^{\circ}$ domains walls that lead to domain boundaries with serrated edges. The micromagnetic simulations, with the inclusion of local strain-fields arising from the microtwin defects, reproduce all the principal building blocks of the entire AF domain structure observed in CuMnAs thin films. Furthermore, the simulations highlight the variety of domain and domain wall structures possible when an array of microtwin defects govern the $\mathrm{AF}$ ordering.

Our results emphasize the sensitivity of AF domain structures to defect structures in thin films. The concentration of such defects may be engineered by varying the growth parameters, particularly the substrate temperature and $\mathrm{Cu} / \mathrm{Mn}$ stoichiometry ${ }^{21}$, providing a mechanism for tailoring the AF domains and domain walls for specific functionality. Introducing some bias during growth, such as substrate stress or miscut angle, may allow further control over the distribution of defect orientations. In terms of optimization of device performance, it was shown previously that the largest electrical readout signals after current pulsing are observed for growth conditions corresponding to the lowest defect densities ${ }^{21}$. This is an indirect indication that the microtwin defects inhibit the nucleation and switching of antiferromagnetic domains. On the other hand, the control of microtwin defects may be beneficial for currentdriven motion of antiferromagnetic domain walls between welldefined pinning centres ${ }^{8}$. Thus, developing high-performance a

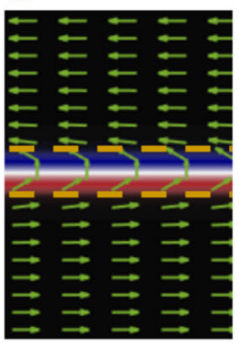

b

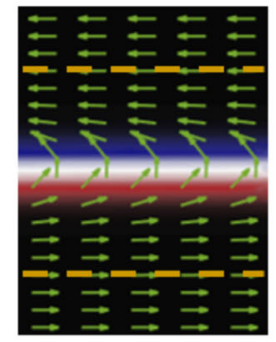

(3)

e

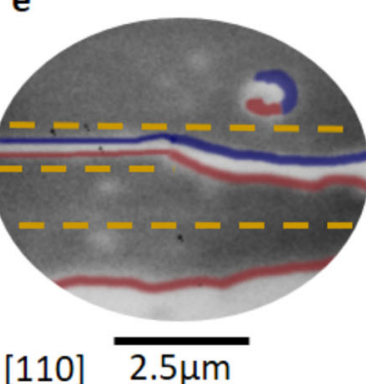

C

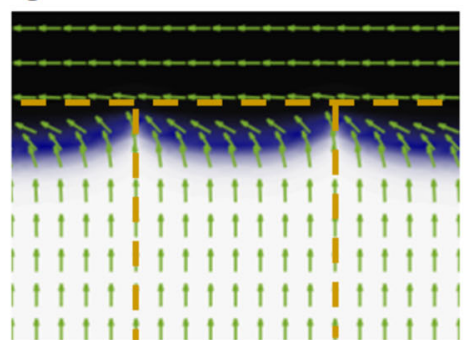

f

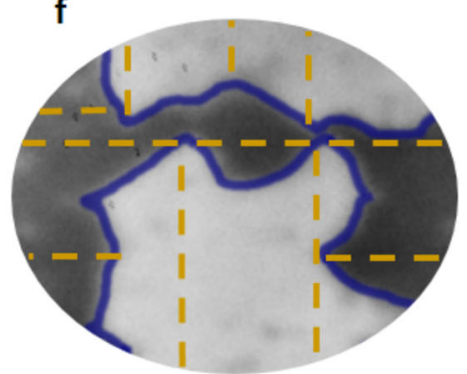

d

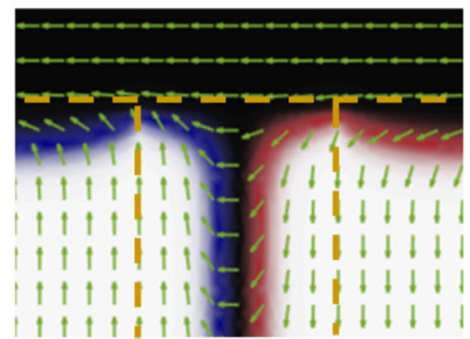

g

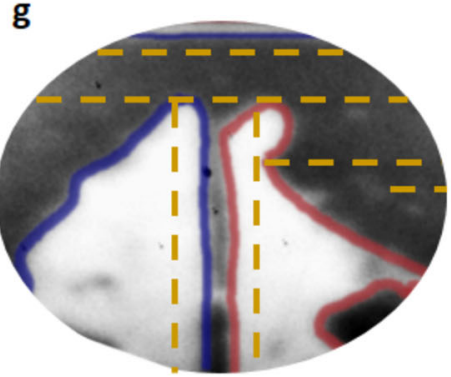

Fig. 4 Micromagnetic simulations. a-d Micromagnetic simulations of the AF domain structure in areas with different microtwin patterns (indicated by the broken yellow lines). a, b Feature parallel microtwins 2 domain wall widths apart (a) and two 7 domain wall widths apart (b). c, d The simulation results for different initial conditions for the same microtwin pattern of one perpendicular and two parallel microtwins forming two T-junctions. The green arrows (in a-d) and color wheel show the local orientation of the Néel vector. e-g XMLD-PEEM images of AF domains overlaid with the microtwin pattern measured in XLD-PEEM (yellow broken lines). 
spintronic devices will rely on a detailed understanding of the nanoscale coupling between the local $\mathrm{AF}$ order and the crystallographic microstructure.

\section{Methods}

Sample fabrication. The $50 \mathrm{~nm}$ CuMnAs films were grown by molecular beam epitaxy on a GaP buffer layer on a $\mathrm{GaP}(001)$ substrate and capped with a $3 \mathrm{~nm} \mathrm{Al}$ film to prevent surface oxidation for the PEEM imaging.

PEEM imaging. The PEEM measurements were performed on beamline I06 at Diamond Light Source, using linearly polarized X-rays incident at $16^{\circ}$ to the film surface. Images were recorded using the ELMITEC-III PEEM on I06 with a hybridpixel electron detector (CheeTah M3 Compact, Amsterdam Scientific Instruments). Magnetic contrast with $\sim 30 \mathrm{~nm}$ spatial resolution was obtained from the asymmetry between images recorded using photon energies corresponding to the maximum and minimum of the $\mathrm{Mn} \mathrm{L}_{3}$ XMLD spectrum (see Supplementary Note 1). The linear polarization (E) was in the plane of the film, and the largest magnetic contrast was obtained between AF domains with spin axes parallel and perpendicular to $\mathbf{E}$. The XMLD spectrum has a similar lineshape, but opposite sign, for $\mathbf{E} \|[110]$ and $\mathbf{E} \|[010]$ (ref. ${ }^{23}$ ). PEEM images with sensitivity to the microtwin configuration were obtained from the asymmetry between images recorded at photon energies corresponding to the maximum and minimum of the $\mathrm{Mn}_{3}$ non-magnetic XLD spectrum (see Supplementary Note 1) with $\mathbf{E}$ at $74^{\circ}$ to the surface. All measurements were performed at room temperature.

Scanning X-ray diffraction microscopy. SXDM was performed on the NanoMAXbeamline at MAX IV Laboratory ${ }^{30,31}$. The beam was focused to a lateral diameter of $100 \mathrm{~nm}$ and the X-ray energy tuned to $10 \mathrm{keV}$. The measurement geometry was determined by three angles: the detector angle $(\delta)$ measured from the direction of the incident beam in the vertical plane, $\Theta$ which defines the angle of incidence with respect to the sample surface in the vertical diffraction plane and $\phi$ which defines the sample azimuth. The sample was scanned laterally over a $2 \mathrm{D}$ mesh at a fixed sample orientation with a diffraction image recorded at each position. The imaging was performed using a Merlin Si Quad area detector with $512 \times 512$ pixels, each $55 \times 55 \mu \mathrm{m}$ in size. The distance between the detector and sample was $0.65 \mathrm{~m}$.

RSMs were constructed via several 2D-mesh scans with a stepsize of $200 \mathrm{~nm}$ at different $\Theta$ angles around the (003) Bragg reflection in $0.02^{\circ}$ increments. For these measurements, $\phi$ was chosen such that the X-ray beam impinged along the CuMnAs [110] direction. Analysis was performed using the xrayutilities toolbox described in reference ${ }^{32}$.

SXDM imaging of the microtwin configuration was performed with $\phi$ chosen so that the beam impinged along the CuMnAs [100] direction. The microtwin configuration was mapped with the sample at an angle $\Delta \Theta= \pm 0.4^{\circ}$ from the Bragg angle. For each angle, the detector plane sliced through two of the microtwinrelated wings in reciprocal space. Consequently, if a microtwin was in the illuminated area, significantly higher intensity was recorded on the corresponding area of the detector, depending on the microtwin orientation. Mapping these areas of high intensity thus revealed the spatial pattern of microtwins with a specific orientation. The SXDM map shown in Fig. $2 \mathrm{c}$ is the sum of SXDM images recorded with $\Theta_{\text {Bragg }}-\Delta \Theta$ and $\Theta_{\text {Bragg }}+\Delta \Theta$. For details see Supplementary Note 3 .

Transmission electron microscopy. For the HAADF-STEM measurements, the CuMnAs samples were capped with an additional $10-15 \mathrm{~nm}$ of carbon ex situ and tungsten in situ. Thin lamellae were prepared by a Ga-focused ion beam. The lamellae were polished at $2 \mathrm{kV}$ and $25 \mathrm{pA}$. The lamellae were investigated using a FEI Titan Themis 60-300 cubed high-resolution (scanning) transmission electron microscope at $300 \mathrm{kV}$. The atomic model overlay shown in Fig. $3 \mathrm{~b}$ was produced using VESTA software ${ }^{33}$.

Micromagnetic simulations. The distribution of the Néel vector $\mathbf{n}(x, y)$ in the presence of different microtwin configurations was simulated using the Matlab PDE TOOlbox to solve the micromagnetic equation

$$
\mathbf{n} \times\left[A \nabla^{2} \mathbf{n}+\mathbf{H}_{\mathbf{n}}\right]=0
$$

with von Neumann boundary conditions. Here $A$ is the magnetic stiffness, $\nabla^{2}$ is the Laplace operator, and

$$
\mathbf{H}_{\mathbf{n}}=-\frac{\partial}{\partial \mathbf{n}}\left(w_{\mathrm{an}}+w_{\mathrm{tw}}+w_{\mathrm{me}}\right),
$$

where $w_{\mathrm{an}}, w_{\mathrm{tw}}$, and $w_{\mathrm{me}}$ are the densities of magnetic anisotropy energy of the bulk film, the magnetic anisotropy energy of the microtwin, and the magnetoelastic energy, respectively. Details can be found in Supplementary Note 4.

\section{Data availability}

The data that support the findings of this study are available within this article and its Supplementary Information.
Received: 4 October 2021; Accepted: 19 January 2022;

Published online: 07 February 2022

\section{References}

1. Jungwirth, T., Marti, X., Wadley, P. \& Wunderlich, J. Antiferromagnetic spintronics. Nat. Nanotechnol. 11, 231-241 (2016).

2. Baltz, V. et al. Antiferromagnetic spintronics. Rev. Mod. Phys. 90, 015005 (2018).

3. Železný, J. et al. Relativistic Néel-order fields induced by electrical current in antiferromagnets. Phys. Rev. Lett. 113, 157201 (2014).

4. Wadley, P. et al. Electrical switching of an antiferromagnet. Science 351 587-590 (2016).

5. Baldrati, L. et al. Mechanism of Néel order switching in antiferromagnetic thin films revealed by magnetotransport and direct imaging. Phys. Rev. Lett. 123, 177201 (2019)

6. Kašpar, Z. et al. Quenching of an antiferromagnet into high resistivity states using electrical or ultrashort optical pulses. Nat. Electron. 4, 30 (2021).

7. Meer, H. et al. Direct imaging of current-induced antiferromagnetic switching revealing a pure thermomagnetoelastic switching mechanism in NiO. Nano Lett. 21, 114 (2021).

8. Wadley, P. et al. Current polarity-dependent manipulation of antiferromagnetic domains. Nat. Nanotechnol. 13, 362-365 (2018).

9. Hubert, A. \& Schäfer, R. Magnetic Domains: The Analysis of Magnetic Microstructures (Springer, 1998).

10. McCord, J. Progress in magnetic domain observation by advanced magnetooptical microscopy. J. Phys. D: Appl. Phys. 48, 333001 (2015).

11. Menon, K. S. R. et al. Surface antiferromagnetic domain imaging using lowenergy unpolarized electrons. Phys. Rev. B 84, 132402 (2011).

12. Scholl, A. et al. Observation of antiferromagnetic domains in epitaxial thin films. Science 287, 1014-1016 (2000).

13. Folven, E. et al. Antiferromagnetic domain reconfiguration in embedded $\mathrm{LaFeO}_{3}$ thin film nanostructures. Nano Lett. 10, 4578-4583 (2010).

14. Bezencenet, O., Bonamy, D., Belkhou, R., Ohresser, P. \& Barbier, A. Origin and tailoring of the antiferromagnetic domain structure in $\alpha-\mathrm{Fe}_{2} \mathrm{O}_{3}$ thin films unraveled by statistical analysis of dichroic spectromicroscopy images. Phys. Rev. Lett. 106, 107201 (2011).

15. Folven, E. et al. Controlling the switching field in nanomagnets by means of domain-engineered antiferromagnets. Phys. Rev. B 92, 094421 (2015).

16. Khymyn, R., Lisenkov, I., Tiberkevich, V., Ivanov, B. A. \& Slavin, A Antiferromagnetic THz-frequency Josephson-like Oscillator Driven by Spin Current. Sci. Rep. 7, 43705 (2017).

17. Gomonay, O., Jungwirth, T. \& Sinova, J. Narrow-band tunable terahertz detector in antiferromagnets via staggered-field and antidamping torques. Phys. Rev. B 98, 104430 (2018).

18. Grzybowski, M. J. et al. Imaging current-induced switching of antiferromagnetic domains in CuMnAs. Phys. Rev. Lett. 118, 057701 (2017).

19. Bodnar, S. Y. et al. Imaging of current induced Néel vector switching in antiferromagnetic $\mathrm{Mn}_{2}$ Au. Phys. Rev. B 99, 140409 (2019).

20. Janda, T. et al. Magneto-Seebeck microscopy of domain switching in collinear antiferromagnet CuMnAs. Phys. Rev. Mater. 4, 094413 (2020).

21. Krizek, F. et al. Molecular beam epitaxy of CuMnAs. Phys. Rev. Mater. 4, 014409 (2020)

22. Krizek, F. et al. Atomically sharp domain walls in an antiferromagnet. Preprint at https://arxiv.org/abs/2012.00894 (2020).

23. Wadley, P. et al. Antiferromagnetic structure in tetragonal CuMnAs thin films. Sci. Rep. 5, 17079 (2015).

24. Wadley, P. et al. Tetragonal phase of epitaxial room-temperature antiferromagnet CuMnAs. Nat. Commun. 4, 2322 (2013).

25. Wadley, P. et al. Antiferromagnetic structure in tetragonal CuMnAs thin films. Sci. Rep. 5, 17079 (2015).

26. Wadley, P. et al. Control of antiferromagnetic spin axis orientation in bilayer Fe/CuMnAs films. Sci. Rep. 7, 11147 (2017).

27. Stangl, J., Mocuta, C., Chamard, V. \& Carbone, D. Nanobeam X-Ray Scattering (John Wiley \& Sons, Ltd, 2013).

28. Pietsch, U., Holy, V. \& Baumbach, T. High-Resolution X-Ray Scattering: From Thin Films to Lateral Nanostructures; Advanced Texts in Physics (Springer New York, 2004).

29. Gomonay, H. \& Loktev, V. M. Magnetostriction and magnetoelastic domains in antiferromagnets. J. Phys. C. 14, 3959-3971 (2002).

30. Bjorling, A. et al. Ptychographic characterization of a coherent nanofocused X-ray beam. Opt. Express 28, 5069-5076 (2020).

31. Johansson, U. et al. NanoMAX: the hard X-ray nanoprobe beamline at the MAXIV Laboratory. J. Synchrotron Rad. 28, 1935-1947 (2021).

32. Kriegner, D., Wintersberger, E. \& Stangl, J. xrayutilities: a versatile tool for reciprocal space conversion of scattering data recorded with linear and area detectors. J. Appl. Crystallogr. 46, 1162-1170 (2013). 
33. Momma, K. \& Izumi, F. VESTA3 for three-dimensional visualization of crystal, volumetric and morphology data. J. Appl. Crystallogr. 44, 1272-1276 (2011).

\section{Acknowledgements}

We thank Diamond Light Source for the allocation of beamtime on beamline I0 6 under Proposal nos. MM22437-1 and NT27146-1. We acknowledge MAX IV Laboratory for beamtime on Beamline NanoMAX under Proposal C20190533. Research conducted at MAX IV, a Swedish national user facility, is supported by the Swedish Research Council under contract 2018-07152, the Swedish Governmental Agency for Innovation Systems under contract 2018-04969, and Formas under contract 2019-02496. CzechNanoLab project LM2018110 funded by MEYS CR is gratefully acknowledged for the financial support of the measurements and sample fabrication at CEITEC Nano Research Infrastructure. D.K., O.G., F.K., V.N., R.P.C., K.A.O., J.S., T.J., P.W., and K.W.E. acknowledge support from the EU FET Open RIA Grant no. 766566. S.R. acknowledges support from Diamond Light Source studentship grant STU0201. V.N., F.K., and T.J. acknowledge support from the Ministry of Education of the Czech Republic Grant No. LM2018110 and LNSM-LNSpin, and the Grant Agency of the Czech Republic Grant No. 19-28375X. O.G. and J.S. acknowledge the ERC Synergy Grant SC2 (No. 610115), the Deutsche Forschungsgemeinschaft (DFG, German Research Foundation) - TRR 173 - 268565370 (projects A03 and B12) and TRR 288 - 422213477 (project A09).

\section{Author contributions}

S.S.D., K.W.E., and P.W. conceived and led the project. S.S.D., K.W.E., and S.R. devised the XMLD-PEEM and XLD-PEEM imaging and performed the measurements with F.M., O.J.A., L.X.B., S.F.P., K.A.O., and P.W. D.C. devised the SXDM experiment and performed the measurements with S.R., D.K., A.B., K.W.E., and S.S.D. D.C. supervised the data analysis, performed by S.R. and D.K., and coordinated the interpretation of the results. O.G. developed the micromagnetic simulations with feedback from S.S.D., K.W.E., and S.R. F.K., J.M., and O.M. performed the HAADF-STEM experiments and data analysis. S.R. combined and led the analysis of the data from the different experimental techniques. R.P.C., F.K., and V.N. fabricated the samples. S.S.D., K.W.E., and S.R. wrote the manuscript with feedback from all authors.

\section{Competing interests}

The authors declare no competing interests.

\section{Additional information}

Supplementary information The online version contains supplementary material available at https://doi.org/10.1038/s41467-022-28311-x.

Correspondence and requests for materials should be addressed to Sonka Reimers, Sarnjeet S. Dhesi or Kevin W. Edmonds.

Peer review information Nature Communications thanks the anonymous reviewer(s) for their contribution to the peer review of this work. Peer reviewer reports are available.

Reprints and permission information is available at http://www.nature.com/reprints

Publisher's note Springer Nature remains neutral with regard to jurisdictional claims in published maps and institutional affiliations.

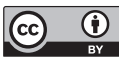

Open Access This article is licensed under a Creative Commons Attribution 4.0 International License, which permits use, sharing, adaptation, distribution and reproduction in any medium or format, as long as you give appropriate credit to the original author(s) and the source, provide a link to the Creative Commons license, and indicate if changes were made. The images or other third party material in this article are included in the article's Creative Commons license, unless indicated otherwise in a credit line to the material. If material is not included in the article's Creative Commons license and your intended use is not permitted by statutory regulation or exceeds the permitted use, you will need to obtain permission directly from the copyright holder. To view a copy of this license, visit http://creativecommons.org/ licenses/by/4.0/.

(C) The Author(s) 2022 